\title{
Magnetic resonance imaging of the ligaments of the craniocervical region at 3Tesla magnetic resonance unit: Quantitative and qualitative assessment
}

\author{
Katerina Vassiou, ${ }^{1,2}$, Kapsalaki Eftichia ${ }^{2}$, Konstantinos Marinos $^{3}$, Filio Kotrogianni ${ }^{3}$, \\ Michail Fanariotis $^{2}$, Ioannis Fezoulidis ${ }^{2}$, Dimitrios Arvanitis ${ }^{1}$ \\ ${ }^{1}$ Department of Anatomy, Medical School, University of Thessalia, Larissa, Greece \\ ${ }^{2}$ Department of Radiology, Medical School, University of Thessalia, Larissa, Greece \\ ${ }^{3}$ Medical School, University of Thessalia, Larissa, Greece \\ Email: avassiou@med.uth.gr
}

Received 8 October 2012; revised 10 November 2012; accepted 19 November 2012

\begin{abstract}
Purpose: The assessment of the morphology and dimensions of the craniocervical ligaments using a 3 Tesla (T) Magnetic Resonance (MR) scanner, the correlatation of our results with those from cadaveric and other MR studies and the detection of the most appropriate sequence for the best imaging of the craniovertebral junction ligaments. Methods: 58 healthy volunteers (mean age 45 years) underwent a Magnetic Resonance Imaging (MRI) of the cervical spine at 3T MR unit. The MRI protocol included axial, coronal and sagittal Proton-Density (PD) sequences and sagittal T1 Fluid Attenuated Inversion Recovery (FLAIR) and $\mathrm{T} 2$ sequences. The images were evaluated by two radiologists and the posterior atlantoocipital ligament, the anterior atlantoocipital ligament, the transverse ligament and the apical ligament were anatomically detected, described and measured. Results: The transverse ligament was identified at $93.1 \%$, the apical ligament was identified at $60.34 \%$, the posterior atlantooccipital membrane was identified at $\mathbf{9 4 . 8 \%}$ and the anterior atlantooccipital membrane was identified at $96.5 \%$ of the cases. All ligaments appeared with low signal intensity, except the anterior atlantooccipital ligament which appeared with intermediate signal intensity. Their length, width and thickness were measured and, in general, correlated well with other anatomic and MR studies. Conclusion: Reliable assessment of the morphology and signal intensity of the craniocervical ligaments can be achieved with PD sequence at 3T MR imaging. The sagittal plane provides better delineation of the craniocervical (CC) ligaments but the axial and coronal planes are of paramount importance in the assessment of the transverse and apical ligaments.
\end{abstract}

Keywords: Craniocervical Region; Ligaments; MRI; Apical Ligament; Transverse Ligament; Atlantooccipital Membrane

\section{INTRODUCTION}

Craniocervical (CC) region's ligaments play an important role in the stability, the mobility and the rotation of the $\mathrm{CC}$ region [1]. The most important ligaments at this region are the transverse ligament, the anterior and posterior atlantooccipital ligament, the apical ligament, the alar ligament and the cruciate ligament (Figure 1). Knowledge of their normal appearance and their variations are of particular importance in determining an injured ligament.

Their anatomy has been described in numerous cadaveric studies and in some anatomical studies using CT and MRI, especially MR units 1.5Tesla (T) [2-11]. Magnetic Resonance Imaging (MRI) is suggested to be the method of choice in the evaluation of the craniocervical (CC) region and its ligaments since it provides high resolution and increased tissue characterization [12-14]. Up to date anatomical MR studies of the craniocervical region have been performed in 1.5T MR units and there has not been set a widely accepted examination protocol in the evaluation of this region. The limitations of $1.5 \mathrm{~T}$ MR units to the detection of thin and closely located structures, such as CC ligaments is already known, compared with 3.0T MR. More specifically, high magnetic field strength MRI systems, as 3T, provide better signal to noise ratio and thus better identification of these small ligaments. To our knowledge there is only one study to date, assessing the anatomy of some of the craniovertebral junction ligaments at 3.0T, called accessory atlantoaxial ligament [11]. In our study we did not concentrate to the detection of these small ligaments, but we 


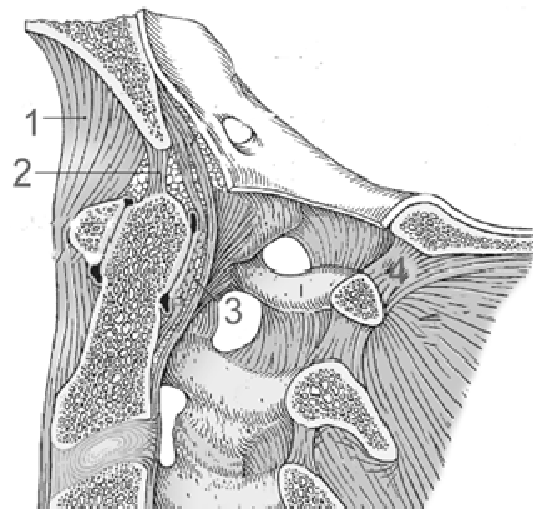

Figure 1. Image illustration of the ligaments at the cranio- cervical region. $1=$ Anterior atlantooccipital ligament, $2=$ Apical ligament, 3 = Transverse ligament, $4=$ Posterior atlantooccipital ligament.

assess the anatomy of the main CC ligaments, such as the anterior and posterior atlantooccipital ligament, the apical ligament and the transverse ligament, using 3T MR unit.

In neck trauma, injury of these ligaments may cause neck pain and may lead to instability and altered mobility in the $\mathrm{CC}$ region. Although it is accepted that the role of MR imaging in severe neck trauma is very important, in minor or moderate neck trauma (acceleration-deceleration collision, whiplash injury) there is a debate concerning the role of MR imaging, especially if there is no neurological deficit [5]. It is very important for the radiologists to be aware of the exact anatomic imaging of these ligaments in order to detect even minor morphological alteration, concerning either their signal intensity or their course, in cases of trauma.

The purpose of this study is the anatomical identification of the ligaments of the $\mathrm{CC}$ region concerning their location, their dimensions and their morphology using a 3T MR scanner, the correlation of our results with those from cadaveric studies and other MR studies and the detection of the most appropriate sequence for the best imaging of these ligaments.

\section{MATERIAL AND METHOD}

In this prospective clinical study, 58 healthy volunteers were included (18 men, 40 women; age range 21 - 50 yrs; mean age 45years) who underwent an MRI of the cervical spine. Written informed consent was obtained from all patients. Inclusion criteria were age between 18 and 50 years without any symptomatology or history of trauma. Exclusion criteria included history of cervical spine or head trauma or systemic inflammatory disease, or other lesion that altered the normal anatomy of the craniocervical region.

The MRI examinations were performed on a 3T sys- tem (signa HDx 3.0T, General Electric Medical Systems, Software Release 15) with a neurovascular 8 channel coil. Subjects were placed in the supine position with their head and neck fixed in a neutral position. Images were obtained in three planes at the level of the craniocervical junction.

The MRI protocol included axial, coronal and sagittal Proton-Density (PD) sequences (TR 2200, TE 56.4, thickness $2 \mathrm{~mm} / 0.2$ gap, time 6:06 $\mathrm{min}$, FOV $24 \times 24$, $416 \times 320,3 \mathrm{NEX})$, sagittal T1 FLAIR sequence (TR 2833, TE 30.7, TI 1057, FOV $24 \times 24$, time 3:11 min, $448 \times 224,2 \mathrm{NEX}$, thickness $2 \mathrm{~mm} / 0.2$ gap) and sagittal T2 sequence. Sagittal and coronal images were obtained with an angle parallel to the predental space and axial images were obtained perpendicular to this line.

The images were evaluated by two radiologists (one with a 10-year subspecialization in imaging of the spine and one with a special interest in neuroanatomy). The posterior atlantoocipital ligament, the anterior atlantoocipital ligament, the transverse ligament and the apical ligament were anatomically detected and described. The length of the anterior and posterior atlantooccipital ligament, the length of the apical ligament, and the height, width and thickness of the transverse ligament were measured. All measurements were performed on a standard PACS workstation. The length of a ligament was defined as the distance between its origin and its insertion. The thickness of the transverse ligament was measured at its mid portion and was defined as the distance between the posterior atlantoaxial joint and the tectorial membrane. The width of the transverse ligament was defined as the distance between it's attachments to the tubercle on either side of the lateral mass of the atlas. The measurements of anterior and posterior atlantoocipital ligament were performed on sagittal PD and T1 images, the measurements of the apical ligament was performed only on the sagittal PD images, as it was better visualized. Moreover, the thickness and height of the transverse ligament were measured on both axial and sagittal PD and T1 images. Transverse ligament width was measured on coronal PD images.

Measurements were correlated with these from other anatomical MR and cadaveric studies.

The signal intensity (SI) of all ligaments was also determined in comparison with the SI of the adjacent structures on the PD images and any signal intensity alterations were described.

Statistical analysis: Paired samples t-test was conducted to compare ligament dimension differences between males and females. The Pearson correlation coefficient was used to calculate possible significant correlations between age and ligament dimensions

\section{RESULTS}

The transverse ligament was clearly defined at 54/58 
cases $(93.1 \%)$ as a broad band of low signal intensity behind the dens on T1-weighted and PD images. It was better identified on the axial and sagittal planes (Figure 2). The mean thickness of the transverse ligament at its midportion was $0.3 \mathrm{~cm}( \pm 0.1)$ (range $0.13 \mathrm{~cm}-0.7 \mathrm{~cm}$ ). The mean craniocaudal height was $1.16 \mathrm{~cm}( \pm 0.2)$ (range $0.8 \mathrm{~cm}-1.61 \mathrm{~cm}$ ). Its width was measured only at 15 cases, as coronal images were not routinely included on our protocol. At these cases transverse ligament mean width was $1.52 \mathrm{~cm}( \pm 0.32)$ (range $1.1 \mathrm{~cm}-2.05 \mathrm{~cm}$ ).

The apical ligament was best defined on sagittal plane on both T1-weighted and PD images, as well on coronal plane, as a thin oblique structure of low signal intensity between the tip of the odontoid process and the anterior margin of the foramen magnum (Figure 3). It was defined at $35 / 58$ subjects $(60.34 \%)$. Its mean craniocaudal length was $0.63 \mathrm{~cm}( \pm 0.15)$ (range $0.38 \mathrm{~cm}-0.99 \mathrm{~cm})$.

The posterior atlantooccipital ligament was best seen on sagittal sections at $55 / 58$ cases $(94.8 \%)$. It was observed as a well-defined structure of low signal intensity both on T1-weighted and PD images situated between the posterior margin of the foramen magnum and the upper margin of the posterior arch of the atlas (Figure 4). The mean craniocaudal length was $0.9 \mathrm{~cm}( \pm 0.21)$ (range $0.56 \mathrm{~cm}-1.62 \mathrm{~cm}$ ).

The anterior atlantooccipital ligament was also best seen on sagittal plane at $56 / 58$ cases $(96.5 \%)$ as a welldefined structure of intermediate signal intensity run between the anterior margin of the foramen magnum and the upper border of the anterior arch of the atlas (Figure 5). The mean craniocaudal lenght was $1.27 \mathrm{~cm}( \pm 0.26)$ (range $0.84 \mathrm{~cm}-1.99 \mathrm{~cm}$ ).

The above observations and measurements are presented on Table 1.

In our study the dimensions of the anterior atlantooccipital, posterior atlantooccipital, apical and transverse ligaments were also evaluated separately in men and women. Their dimensions are depicted on Table 2 and the correlation between ligaments dimensions and gender is shown on Table 3. There was a significant difference between men and women in the apical ligament length ( $t$-score $-2.65, p=0.012)$. There was no correlation between age and ligament measurement.

\section{DISCUSSION}

MRI is the method of choice for assessing the complex anatomy of the $\mathrm{CC}$ junction ligaments, due to multiplanar high resolution images. The introduction of high field strength (3T) MR scanners has greatly improved the delineation of the CC structures $[5,8,15]$. Increased field strengths permit higher signal-to-noise ratio (SNR) as well as spatial resolution and thus better delineation of small structures, allowing thinner slices with excellent
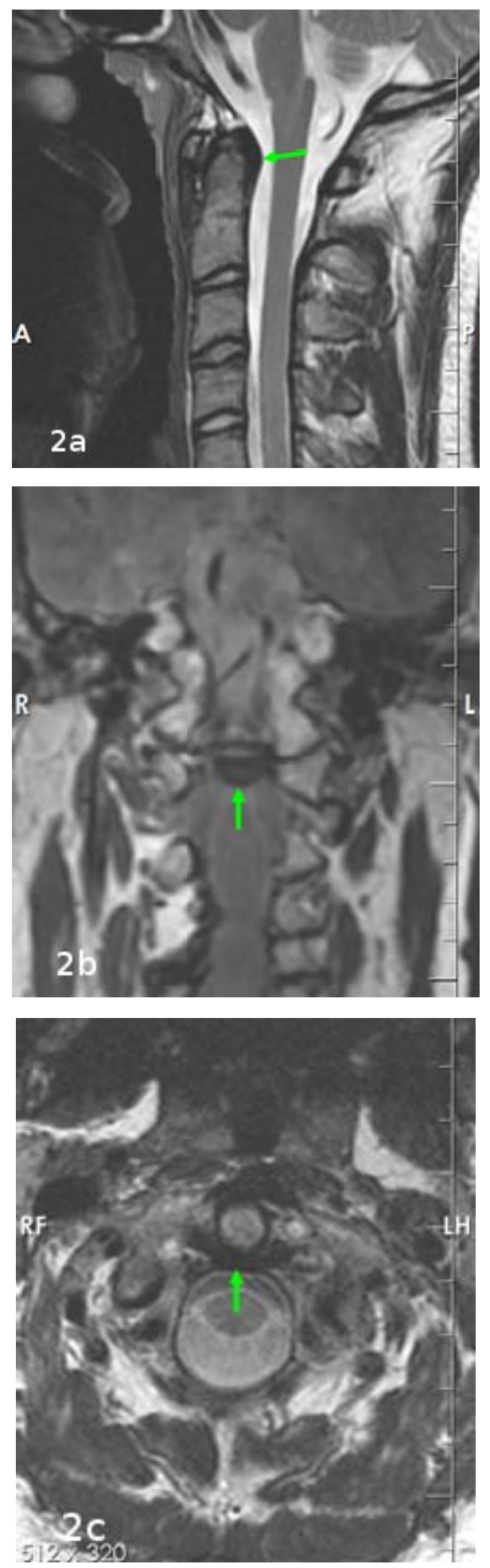

Figure 2. (a) Sagittal; (b) Coronal and (c) Axial PD images of the transverse ligament. (a) The transverse ligament is clearly seen as a low signal intensity structure behind the dens (arrow); (b) The low signal intensity transverse liga- ment, part of the cruciate ligament (arrow); (c) Wellde- fined low signal intensity ligament around the dens (arrow). 


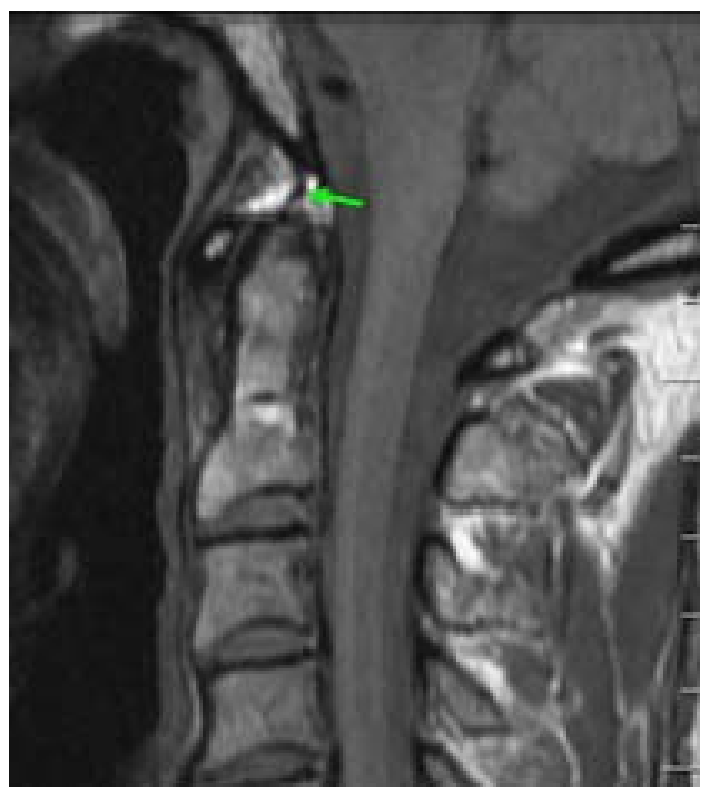

Figure 3. Sagittal PD image of the atlantoaxial joint. The apical ligament is a well-defined thin low signal intensity structure between the tip of the odontoid process and the anterior margin of the foramen magnum (arrow).

signal to noise ratio in a short time [16-18].

There is a number of studies in the literature on imaging of normal anatomy and imaging techniques for the transverse, apical, anterior and posterior atlantooccipital ligament $[2,5-8,15,19-21]$. The majority of these studies have been carried out on 1.5T MR units and there is only one study assessing the normal anatomy of accessory atlantoaxial ligament at $3 \mathrm{~T}$ and its relationship to the ligamentous complex of the CC junction [11]. However, there are a number of published studies focusing on imaging of injuries of these ligaments [20,22-25]. In our study a $3 T$ MR scanner was used for the identification and characterization of some of the CC ligaments in a normal population. These ligaments are very important at the motor function of the $\mathrm{CC}$ junction and the exact knowledge of their appearance is significant, especially in cases of neck trauma.

T1, T2 and PD sequences may be used for the identification of spinal ligaments. Normal ligaments, due to their fibrous nature, appear as low signal intensity structures on all sequences $[5,6]$. T1-weighted sequence, which is considered an anatomical sequence, better identifies the general bony anatomy of the $\mathrm{CC}$ region and the larger ligaments of the spine, as the anterior and posterior longitudinal ligaments and the ligamentum flavum, but present lower specificity in the identification of smaller ligaments, as the $\mathrm{CC}$ junction ligaments $[5,6]$. In our study, we were based on the T2 and PD sequences for the $\mathrm{r}$ of the $\mathrm{CC}$ ligaments. These sequences provide high contrast between anatomical structures and are quite
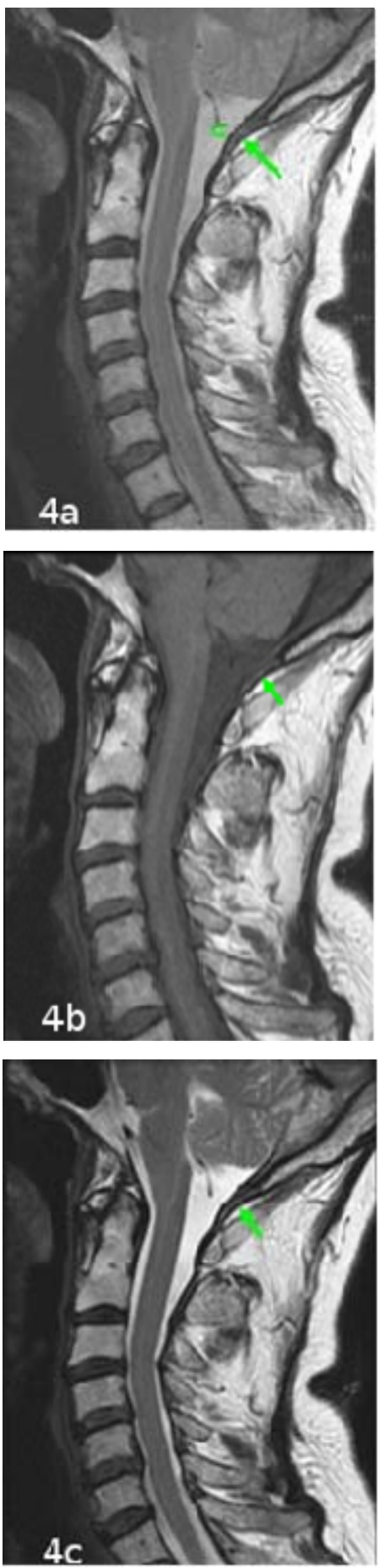

Figure 4. (a) Sagittal PD, (b) T1 and (c) T2-weighted images of the atlantoaxial joint. The posterior antlantooccipital ligament is well- defined as a broad, thin ligament between the posterior arch of the atlas and the posterior rim of the foramen magnum (arrow). In our study, in most cases, it is easily distinguished from the posterior dura mater (curved arrow) of the vertebral canal. 
Table 1. Observations on the ligaments under investigation in the craniocervical junction.

\begin{tabular}{|c|c|}
\hline Transverse ligament & $\begin{array}{l}\text { Better visualized on axial and sagittal planes; low signal intensity on T1 and } \\
\text { PD-weighted sequences; mean thickness } 0.3 \mathrm{~cm}( \pm 0.1) \text {; mean craniocaudal height } \\
1.16 \mathrm{~cm}( \pm 0.2) \text {; mean width } 1.52 \mathrm{~cm}( \pm 0.32)\end{array}$ \\
\hline $\begin{array}{l}\text { Anterior atlantooccipital } \\
\text { ligaments }\end{array}$ & $\begin{array}{l}\text { Better visualized on sagittal plane; intermediate signal intensity on T1 and } \\
\text { PD-weighted sequences; mean craniocaudal height } 1.27 \mathrm{~cm}( \pm 0.26)\end{array}$ \\
\hline $\begin{array}{l}\text { Posterior atlantooccipital } \\
\text { ligaments }\end{array}$ & $\begin{array}{l}\text { Better visualized on sagittal plane; low signal intensity on T1 and T2-weighted } \\
\text { sequences; mean craniocaudal height } 0.9 \mathrm{~cm}( \pm 0.21)\end{array}$ \\
\hline Apical ligament & $\begin{array}{l}\text { Better visualized on sagittal plane; low signal intensity on T1 and T2-weighted } \\
\text { sequences; mean craniocaudal length } 0.63 \mathrm{~cm}( \pm 0.15)\end{array}$ \\
\hline
\end{tabular}

Table 2. Ligaments dimensions in men and women.

\begin{tabular}{|c|c|c|c|c|c|c|c|}
\hline & & $\begin{array}{c}\text { Anterior } \\
\text { atlantooccipital }\end{array}$ & $\begin{array}{c}\text { Posterior } \\
\text { atlantooccipital }\end{array}$ & Apical & $\begin{array}{l}\text { Transverse } \\
\text { (height) }\end{array}$ & $\begin{array}{l}\text { Transverse } \\
\text { (thickness) }\end{array}$ & $\begin{array}{l}\text { Transverse } \\
\text { (width) }\end{array}$ \\
\hline \multirow{2}{*}{ Women } & $\begin{array}{l}\text { Mean } \\
( \pm \text { SD })\end{array}$ & $1.24( \pm 0.26)$ & $0.9( \pm 0.22)$ & $\begin{array}{c}0.58 \\
( \pm 0.12)\end{array}$ & $1.14( \pm 0.2)$ & $0.29( \pm 0.12)$ & $1.5( \pm 0.35)$ \\
\hline & Min Max & 0.841 .86 & 0.561 .62 & 0.380 .86 & 0.81 .61 & 0.130 .7 & 1.132 .05 \\
\hline \multirow{2}{*}{ Men } & $\begin{array}{l}\text { Mean } \\
( \pm \mathrm{SD})\end{array}$ & $1.33( \pm 0.27)$ & $0.91( \pm 0.22)$ & $\begin{array}{c}0.71 \\
( \pm 0.17)\end{array}$ & $1.21( \pm 0.19)$ & $0.31( \pm 0.06)$ & $1.56( \pm 0.28)$ \\
\hline & Min Max & 0.941 .99 & 0.651 .33 & 0.470 .99 & 0.891 .51 & 0.220 .43 & 1.11 .8 \\
\hline \multirow{2}{*}{ Total } & $\begin{array}{l}\text { Mean } \\
( \pm \mathrm{SD})\end{array}$ & $1.27( \pm 0.26)$ & $0.9( \pm 0.21)$ & $\begin{array}{c}0.63 \\
( \pm 0.15)\end{array}$ & $1.16( \pm 0.2)$ & $0.3( \pm 0.1)$ & $1.52( \pm 0.32)$ \\
\hline & Min Max & 0.841 .99 & 0.561 .62 & 0.380 .99 & 0.81 .61 & 0.130 .7 & 1.12 .05 \\
\hline
\end{tabular}

Table 3. Correlation between ligaments dimensions and gender: significant difference between men and women in the apical ligament length was shown.

\begin{tabular}{ccccccc}
\hline & Anterior atlantooccipital & $\begin{array}{c}\text { Posterior } \\
\text { atlantooccipital }\end{array}$ & Apical & $\begin{array}{c}\text { Transverse } \\
\text { (height) }\end{array}$ & $\begin{array}{c}\text { Transverse } \\
\text { (thickness) }\end{array}$ & Transverse (width) \\
\hline t-test & -1.171 & -0.2131 & -2.6473 & -1.2674 & -0.6576 & -0.3291 \\
p value & 0.24 & 0.83 & 0.012 & 0.21 & 0.513 & 0.747 \\
\hline
\end{tabular}

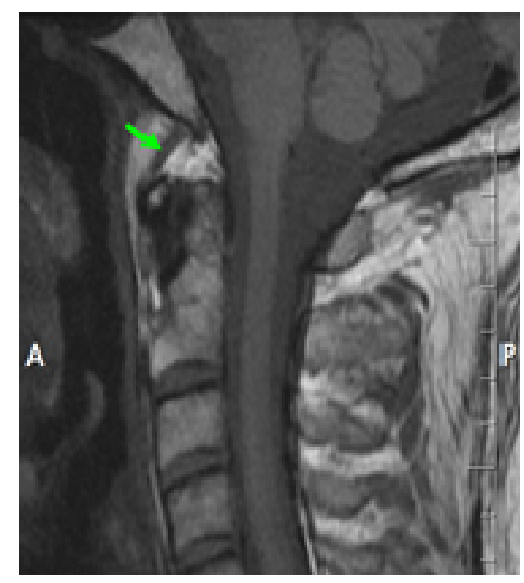

Figure 5. Sagittal T1 image of the atlantoaxial joint. Ante- riorly, the anterior atlantooccipital ligament is clearly seen as a well-defined low signal intensity structure which ex- tends between the upper part of the anterior arch of the atlas to the foramen magnum. sufficient to delineate the ligaments from the surrounding tissues, like fat, muscle and cerebrospinal fluid [5] and to better identify their pathology [5,6]. PD sequence offers increased contrast resolution due to low echo time and it provides better delineation of these ligaments from the surrounding soft tissues. In a previous study of Krakenes et al. [24] it is referred that compared with 2D T2weighted images, 2D PD-weighted sequences provided better discrimination between the ligaments and surrounding tissues because of a higher signal-to-noise ratio, but with the limitation of increased acquisition time (approximately $30 \mathrm{~min}$ ). In our study $\mathrm{PD}$-weighted sequence lasts 6:06 $\mathrm{min}$ due to the increased magnetic field strength of $3 \mathrm{~T}$, which permits acquisition of thin slices at short acquisition times. Our results were mainly based on PD images performed on the sagittal and coronal planes. The axial plane was mainly used for the evaluation of the transverse ligament. T2 and T1 sequences were used complimentarily to the PD sequences.

The apical ligament is placed between the tip of the 
odontoid process and the anterior margin of the foramen magnum in front of the cruciform ligament (Figure 1) [26]. The apical ligament was better visualized on sagittal planes and has minimal significance in the stability of $\mathrm{CC}$ region [27]. In our study, the mean length of the apical ligament was $0.63 \mathrm{~cm}( \pm 0.15)$ which is in agreement with the cadaveric study of Tubbs et al. [21] although other studies $[23,28]$ report greater length of the apical ligament. We identified the apical ligament only in 35 out of 58 subjects $(60.34 \%)$, ratio slightly higher than that reported in the published literature [2]. In the remaining subjects ( 23 cases) the ligament was not identified due to inability to differentiate it from the adjacent structures like fat and venous plexus in 8 cases (Figure 6) and due to the imaging plane of our sagittal and coronal images, which was not parallel to it, at the remaining 14 cases [7,21]. These are in agreement with published data reporting absence of the apical ligament in some individuals [21] or mis-distinctness from adjacent fat pad and venous plexus [7,21].

The transverse ligament of the atlas is a thick semicircular band with arcuate course across the ring of the atlas, attached on either side to a small tubercle on the medial surface of the lateral mass of the atlas (Figure 1). Its shape is concave in front, it is broader and thicker in the middle than at the ends and it helps maintain the odontoid process in contact with the anterior arch [26]. As it crosses the odontoid process, two small fasciculi derived from the superior and inferior fibers of the ligament are extended upwards and downwards, called crus superius and crus inferius. The crus superius is attached to the lower margin of the occipital bone and the crus inferius is attached to the posterior surface of the body of the

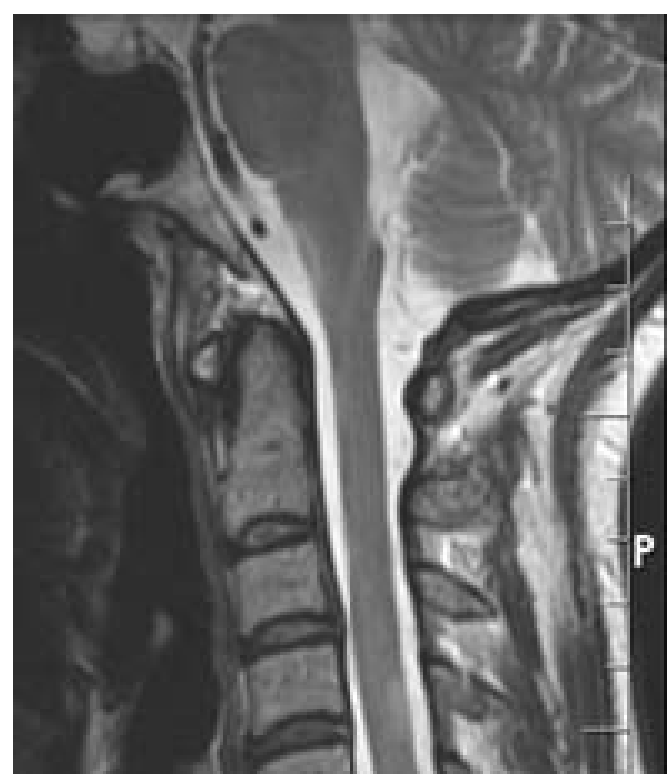

Figure 6. Sagittal T2 image of the atlantoaxial joint: the apical ligament is not clearly seen. axis. The aforementioned complex forms the cruciate ligament of the atlas [26]. The transverse ligament is the most significant ligament for the stability between the head and neck at the atlantoaxial joint [29]. In our study, the transverse ligament was identified in 54 out of 58 subjects $(93.1 \%)$, which is in accordance with the published literature $[2,5]$. The cause for the non delineation of the transverse ligament was degenerative changes at the region. In our study, the mean thickness at its midportion was $0.3 \mathrm{~cm}( \pm 0.1)$ which is partially in agreement with-published data [5] whereas another study reports its thickness to be bigger. The mean craniocaudal height of the transverse ligament was $1.16 \mathrm{~cm}( \pm 0.2)$. In the published studies performed with MR, this measurements is reported to be close to our measurements [5,30], in cadaveric studies the length of the transverse ligament is reported to be longer [30-32]. The transverse ligament was depicted adequately on all planes, axial, coronal and sagittal, both at T1 and PD sequences, a fact that is in agreement with published data. The plane one uses depends on the side the transverse ligament that has to be checked.

The anterior atlantooccipital ligament extends between the anterior margin of the foramen magnum and the upper border of the anterior arch of the atlas (Figure 1). The posterior atlantooccipital ligament extends between the posterior margin of the foramen magnum and the upper border of the posterior arch of the atlas (Figure 1) [26]. It joins with the posterior dura mater of the vertebral canal and, sometimes, its distinction from the dura matter is difficult, especially on sagittal images [20,33]. However, in our study this distinction was possible in most cases both in T2 and PD sequences, due to the increase resolution of 3T (Figure 4). Concerning the functional significance of the anterior and posterior atlantooccipital ligaments, a cadaveric study has been shown that sectioning of the anterior or posterior atlantooccipital ligament alone did not make the neck unstable [34]. The anterior atlantooccipital ligament was not defined in 2 cases $(3.4 \%)$ since it was indistinguishable from the adjacent structures on mid-sagittal images. The posterior atlantooccipital ligament was not defined clearly in 3 cases $(5.1 \%)$ because of hyperextension of the neck. In our study, the detection of anterior atlantooccipital ligament was highly satisfactory which is in contrast with a publication [5] indicating poor visualization of the anterior atlantooccipital ligament being obscured from the surrounding fat. Concerning the posterior atlantooccipital ligament our results are significantly better than in other publications [5]. The mean craniocaudal lenght of the anterior antlantooccipital ligament was $1.27 \mathrm{~cm}( \pm 0.26)$ and the mean craniocaudal lenght of the posterior antlantooccipital ligament was $0.9 \mathrm{~cm}( \pm 0.21)$.

Normal ligaments show low signal intensity on all se- 
quences. However, it has been reported that some ligaments may show higher signal intensity. This finding is usual after trauma, but it is also reported in non-injured individuals $[6,25,35]$. Some of the most known causes of high-signal in tendons and ligaments are age-dependent degenerative changes and the presence of fat tissue disseminated among their fibres [9]. The latter might represent a normal variation in the ligament's structure, although it has not been histologically proven [9]. Schweitzer et al. [8] have reported that the anterior atlantooccipital ligament was found to be of higher signal intensity than the other ligaments of the CC junction. In our study, the anterior atlantooccipital ligament appeared with high signal on T2 and PD images (Figure 7(a)) in 15 cases $(25 \%)$ and it was not age-dependant. More specifically, it was defined in eight individuals aged $25-40$ years old, four aged 40 - 50 years and in three individuals older than 50 years old. Increased signal intensity was
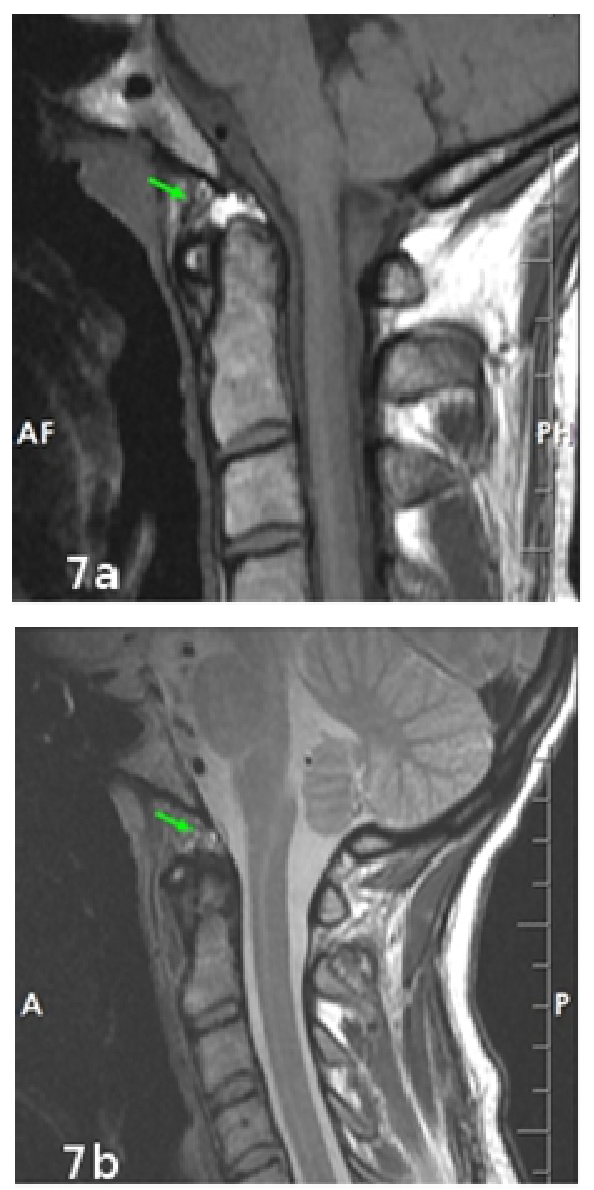

Figure 7. (a) Sagittal T1 image of the atlantoaxial joint: High signal in the medial part of the anterior atlantooccipital ligament is depicted (arrow); (b) Sagittal PD image of the atlantoaxial joint: Heterogenous partially high signal is seen in the posterior part of the apical ligament (arrow). also observed at the apical ligament in 6 cases (Figure 7(b)) and at the transverse ligament in one case. We were not able to demonstrate an association between such changes and the age of these individuals.

In conclusion, reliable assessment of the morphology and signal intensity of the CC ligaments can be achieved with $3 \mathrm{~T}$ MR imaging. The sagittal plane provides better delineation of the $\mathrm{CC}$ ligaments, but the axial and coronal planes are of paramount importance in the assessment of the transverse and apical ligaments. The PD sequences provide excellent identification of the ligaments and their signal intensity. Knowledge of the normal measurements and the normal variations of the $\mathrm{CC}$ junction ligaments is of paramount importance for the assessment of pathology in this area, particularly after trauma. Moreover, 3T MRI provides excellent identification of these ligaments, using thin, high resolution images, obtained in a short acquisition time.

\section{REFERENCES}

[1] Daniels, D.L., Williams, A.L. and Haughton, V.M. (1983) Computed tomography of the articulations and ligaments at the occipito-atlantoaxial region. Radiology, 146, 709716

[2] Baumert, B., Wortler, K., Steffinger, D., Schmidt, G.P., Reiser, M.F. and Baur-Melnyk, A. (2009) Assessment of the internal craniocervical ligaments with a new magnetic resonance imaging sequence: Three-dimensional turbo spin echo with variable flip-angle distribution (SPACE). Magnetic Resonance Imaging, 27, 954-960. doi:10.1016/j.mri.2009.01.012

[3] Dvorak, J. and Panjabi, M.M. (1987). Functional anatomy of the alar ligaments. Spine, 12, 183-189. doi:10.1097/00007632-198703000-00016

[4] Kim, H.J., Jun, B.Y., Kim, W.H., Cho, Y.K., Lim, M.K. and Suh, C.H. (2002) MR imaging of the alar ligament: Morphologic changes during axial rotation of the head in asymptomatic young adults. Skeletal Radiology, 31, $637-$ 642. doi:10.1007/s00256-002-0572-2

[5] Krakenes, J., Kaale, B.R., Rorvik, J. and Gilhus, N.E. (2001) MRI assessment of normal ligamentous structures in the craniovertebral junction. Neuroradiology, 43, 10891097. doi: $10.1007 / \mathrm{s} 002340100648$

[6] Pfirrmann, C.W., Binkert, C.A., Zanetti, M., Boos, N. and Hodler, J. (2001) MR morphology of alar ligaments and occipitoatlantoaxial joints: Study in 50 asymptomatic subjects. Radiology, 218, 133-137.

[7] Saifuddin, A., Green, R. and White, J. (2003) Magnetic resonance imaging of the cervical ligaments in the absence of trauma. Spine, 28, 1686-1691.

[8] Schweitzer, M.E., Hodler, J., Cervilla, V. and Resnick, D. (1992) Craniovertebral junction: Normal anatomy with MR correlation. American Journal of Roentgenology, 158, 1087-1090.

[9] Vetti, N., Krakenes, J., Eide, G.E., Rorvik, J., Gilhus, N.E. 
and Espeland, A. (2009) MRI of the alar and transverse ligaments in whiplash-associated disorders (WAD) grades 1-2: High-signal changes by age, gender, event and time since trauma. Neuroradiology, 51, 227-235. doi:10.1007/s00234-008-0482-7

[10] Willauschus, W.G., Kladny, B., Beyer, W.F., Gluckert, K., Arnold, H. and Scheithauer, R. (1995) Lesions of the alar ligaments. In vivo and in vitro studies with magnetic resonance imaging. Spine, 20, 2493-2498. doi:10.1097/00007632-199512000-00006

[11] Yuksel, M., Heiserman, J.E. and Sonntag, V.K. (2006) Magnetic resonance imaging of the craniocervical junction at 3-T: Observation of the accessory atlantoaxial ligaments. Neurosurgery, 59, 888-892.

[12] Bagley, L.J. (2006). Imaging of spinal trauma. Radiologic Clinics of North America, 44, 1-12. doi:10.1016/j.rcl.2005.08.004

[13] Stabler, A., Eck, J., Penning, R., Milz, S.P., Bartl, R., Resnick, D. and Reiser, M. (2001) Cervical spine: Postmortem assessment of accident injuries - comparison of radiographic, MR imaging, anatomic, and pathologic findings. Radiology, 221, 340-346. doi:10.1148/radiol.2212010336

[14] Van Geothem, J.W., Biltjes, I.G., van den Hauwe, L., Parizel, P.M. and De Schepper, A.M. (1996) Whiplash injuries: Is there a role for imaging? European Journal of Radiology, 22, 30-37. doi:10.1016/0720-048X(95)00696-N

[15] Ellis, J.H., Martel, W., Lillie, J.H. and Aisen, A.M. (1991) Magnetic resonance imaging of the normal craniovertebral junction. Spine, 16, 105-111.

[16] Ashman, C.J., Farooki, S., Abduljalil, A.M. and Chakeres, D.W. (2002). In vivo high resolution coronal MRI of the wrist at 8.0 tesla. Journal of Computer Assisted Tomography, 26, 387-391. doi:10.1097/00004728-200205000-00013

[17] Farooki, S., Ashman, C.J., Yu, J.S., Abduljalil, A. and Chakeres, D. (2002) In vivo high-resolution MR imaging of the carpal tunnel at 8.0 tesla. Skeletal Radiology, 31, 445-450. doi:10.1007/s00256-002-0506-Z

[18] Saupe, N., Prussmann, K.P., Luechinger, R., Bosiger, P., Marincek, B. and Weishaupt, D. (2005) MR imaging of the wrist: Comparison between 1.5- and 3-T MR imaging - preliminary experience. Radiology, 234, 256-264. doi:10.1148/radiol.2341031596

[19] Engelman, E.D., Schnitzlein, S.H., Hilbelink, D.R., Murtagh, F.R. and Silbiger, M.L. (1989) Imaging anatomy of the cranio-vertebral junction (occipito-atlanto-axial joint). Clinical Anatomy, 2, 241-252. doi:10.1002/ca.980020405

[20] Krakenes, J., Kaale, B.R., Nordli, H., Moen, G., Rorvik, J. and Gilhus, N.E. (2003) MR analysis of the transverse ligament in the late stage of whiplash injury. Acta Radiologica, 44, 637-644.

[21] Tubbs, R.S., Grabb, P., Spooner, A., Wilson, W., and Oakes, W.J. (2000). The apical ligament: anatomy and functional significance. Journal of Neurosurgery, 92, 197-200.
[22] Grabb, B.C., Frye, T.A., Hedlund, G.L., Vaid, Y.N., Grabb, P.A. and Royal, S.A. (1999) MRI diagnosis of suspected atlanto-occipital dissociation in childhood. Pediatric Radiology, 29, 275-281. doi: $10.1007 / \mathrm{s} 002470050588$

[23] Hecker, P. (1922) Appareil ligamenteux occipito-atloïdoaxoïdien: Étude d'anatomie comparée. Anatomy, Cytology, Histology, Histopathology, 1, 417-433.

[24] Krakenes, J., Kaale, B.R., Moen, G., Nordli, H., Gilhus, N.E. and Rorvik, J. (2002) MRI assessment of the alar ligaments in the late stage of whiplash injury: A study of structural abnormalities and observer agreement. Neuroradiology, 44, 617-624. doi:10.1007/s00234-002-0799-6

[25] Myran, R., Kvistad, K.A., Nygaard, O.P., Andresen, H., Folvik, M. and Zwart, J.A. (2008) Magnetic resonance imaging assessment of the alar ligaments in whiplash injuries: A case-control study. Spin, 33, 2012-2016. doi:10.1097/BRS.0b013e31817bb0bd

[26] Williams, P.L.W.R., Dyston, M. and Banister, L.H. (1989) In Gray's anatomy. 37th Edition, Churchill-Liv- ingstone, Edinburgh.

[27] Tubbs, R.S., Hallock, J.D., Radcliff, V., Naftel, R.P., Mortazavi, M., Shoja, M.M., Loukas, M. and CohenGadol, A.A. (2011) Ligaments of the craniocervical junction. Journal of Neurosurgery: Spine, 14, 697-709. doi:10.3171/2011.1.SPINE10612

[28] Panjabi, M.M., Oxland, T.R. and Parks, E.H. (1991) Quantitative anatomy of cervical spine ligaments. Part II. Middle and lower cervical spine. Journal of Spinal Disorders, 4, 277-285.

doi:10.1097/00002517-199109000-00004

[29] Tubbs, R.S., Kelly, D.R., Humphrey, E.R., Chua, G.D., Shoja, M.M., Salter, E.G., Acakpo-Satchivi, L., Wellons, J.C., Blount, J.P. and Oakes, W.J. (2007) The tectorial membrane: Anatomical, biomechanical, and histological analysis. Clinical Anatomy, 20, 382-386. doi:10.1002/ca.20334

[30] Cattrysse, E., Barbero, M., Kool, P., Gagey, O., Clarys, J.P. and Van Roy, P. (2007) 3D morphometry of the transverse and alar ligaments in the occipito-atlanto-axial complex: An in vitro analysis. Clinical Anatomy, 20, 892898. doi:10.1002/ca.20559

[31] Okazaki, K. (1995) Anatomical study of the ligaments in the occipito-atlantoaxial complex. Nihon Seikeigeka Gakkai Zasshi, 69, 1259-1267.

[32] Panjabi, M.M., Crisco, J.J., Lydon, C. and Dvorak, J. (1998) The mechanical properties of human alar and transverse ligaments at slow and fast extension rates. Clinical Biomechanics, 13, 112-120. doi:10.1016/S0268-0033(97)00053-3

[33] Soames, R. (1995) Skeletal system. In: Williams, P.L., Bannister, L.H., Berry, M.M., et al., Eds., Gray's Anatomy, 38th Edition, Churchill Livingstone, New York, 425-736.

[34] Harris, M.B., Duval, M.J., Davis Jr., J.A. and Bernini, P.M. (1993) Anatomical and roentgenographic features of atlantooccipital instability. Journal of Spinal Disorders, 6, 5-10. doi:10.1097/00002517-199302000-00002 
[35] Roy, S., Hol, P.K., Laerum, L.T. and Tillung, T. (2004) Pitfalls of magnetic resonance imaging of alar ligament.
Neuroradiology, 46, 392-398. doi:10.1007/s00234-004-1193-3 